\title{
CODES OVER RINGS OF SIZE FOUR, HERMITIAN LATTICES, AND CORRESPONDING THETA FUNCTIONS
}

\author{
T. SHASKA AND G. S. WIJESIRI
}

(Communicated by Wen-Ching Winnie Li)

\begin{abstract}
Let $K=Q(\sqrt{-\ell})$ be an imaginary quadratic field with ring of integers $\mathcal{O}_{K}$, where $\ell$ is a square free integer such that $\ell \equiv 3 \bmod 4$, and let $C=[n, k]$ is a linear code defined over $\mathcal{O}_{K} / 2 \mathcal{O}_{K}$. The level $\ell$ theta function $\Theta_{\Lambda_{\ell}(C)}$ of $C$ is defined on the lattice $\Lambda_{\ell}(C):=\left\{x \in \mathcal{O}_{K}^{n}: \rho_{\ell}(x) \in C\right\}$, where $\rho_{\ell}: \mathcal{O}_{K} \rightarrow \mathcal{O}_{K} / 2 \mathcal{O}_{K}$ is the natural projection. In this paper, we prove that:

i) for any $\ell, \ell^{\prime}$ such that $\ell \leq \ell^{\prime}, \Theta_{\Lambda_{\ell}}(q)$ and $\Theta_{\Lambda_{\ell^{\prime}}}(q)$ have the same coefficients up to $q^{\frac{\ell+1}{4}}$,

ii) for $\ell \geq \frac{2(n+1)(n+2)}{n}-1, \Theta_{\Lambda_{\ell}}(C)$ determines the code $C$ uniquely,

iii) for $\ell<\frac{2(n+1)(n+2)}{n}-1$, there is a positive dimensional family of symmetrized weight enumerator polynomials corresponding to $\Theta_{\Lambda_{\ell}}(C)$.
\end{abstract}

\section{INTRODUCTION}

Let $K=Q(\sqrt{-\ell})$ be an imaginary quadratic field with ring of integers $\mathcal{O}_{K}$, where $\ell$ is a square free integer such that $\ell \equiv 3 \bmod 4$. Then the image $\mathcal{O}_{K} / 2 \mathcal{O}_{K}$ of the projection $\rho_{\ell}: \mathcal{O}_{K} \rightarrow \mathcal{O}_{K} / 2 \mathcal{O}_{K}$ is $\mathbb{F}_{4}\left(\right.$ resp., $\left.\mathbb{F}_{2} \times \mathbb{F}_{2}\right)$ if $\ell \equiv 3 \bmod 8$ (resp., $\ell \equiv 7 \bmod 8$ )

Let $\mathcal{R}$ be a ring isomorphic to $\mathbb{F}_{4}$ or $\mathbb{F}_{2} \times \mathbb{F}_{2}$ and $C=[n, k]$ be a linear code over $\mathcal{R}$ of length $n$ and dimension $k$. An admissible level $\ell$ is an $\ell$ such that $\ell \equiv 3$ $\bmod 8$ if $\mathcal{R}$ is isomorphic to $\mathbb{F}_{4}$ or $\ell \equiv 7 \bmod 8$ if $\mathcal{R}$ is isomorphic to $\mathbb{F}_{2} \times \mathbb{F}_{2}$. Fix an admissible $\ell$ and define $\Lambda_{\ell}(C):=\left\{x \in \mathcal{O}_{K}^{n}: \rho_{\ell}(x) \in C\right\}$.

Then, the level $\ell$ theta function $\Theta_{\Lambda_{\ell}(C)}(\tau)$ of the lattice $\Lambda_{\ell}(C)$ is given as the symmetric weight enumerator swe $_{C}$ of $C$, evaluated on the theta functions defined on cosets of $\mathcal{O}_{K} / 2 \mathcal{O}_{K}$. In this paper we study the following two questions:

i) How do the theta functions $\Theta_{\Lambda_{\ell}(C)}(\tau)$ of the same code $C$ differ for different levels $\ell$ ?

ii) Can nonequivalent codes give the same theta functions for all levels $\ell$ ?

In an attempt to study the second question, Chua in [1] gives an example of two nonequivalent codes that give the same theta function for level $\ell=7$ but not for higher level thetas. We will show in this paper how such an example is not a coincidence. Our main results are as follows:

Received by the editors January 10, 2007 and, in revised form, February 14, 2007, February 21, 2007, and February 24, 2007.

2000 Mathematics Subject Classification. Primary 11H71, 94B75; Secondary 11H31.

Key words and phrases. Theta functions, Hermitian lattices, codes. 
Theorem 1. Let $C$ be a code defined over $R$. For all admissible $\ell, \ell^{\prime}$ such that $\ell>\ell^{\prime}$, the following holds:

$$
\Theta_{\Lambda_{\ell}}(C)=\Theta_{\Lambda_{\ell^{\prime}}}(C)+\mathcal{O}\left(q^{\frac{\ell^{\prime}+1}{4}}\right) .
$$

Theorem 2. Let $C$ be a code of size $n$ defined over $\mathcal{R}$ and $\Theta_{\Lambda_{\ell}}(C)$ be its corresponding theta function for level $\ell$. Then the following hold:

i) For $\ell<\frac{2(n+1)(n+2)}{n}-1$, there is a $\delta$-dimensional family of symmetrized weight enumerator polynomials corresponding to $\Theta_{\Lambda_{\ell}}(C)$, where $\delta \geq \frac{(n+1)(n+2)}{2}-\frac{n(\ell+1)}{4}-1$.

ii) For $\ell \geq \frac{2(n+1)(n+2)}{n}-1$ and $n<\frac{\ell+1}{4}$, there is a unique symmetrized weight enumerator polynomial which corresponds to $\Theta_{\Lambda_{\ell}}(C)$.

This paper is organized as follows. In the second section, we give a basic introduction of lattices and theta functions. We define a lattice $\Lambda$ over a number field $K$ in general, the theta series of a lattice, and the one-dimensional theta series and its shadow. Then we discuss the lattices over imaginary quadratic fields $K=Q(\sqrt{-\ell})$ with a ring of integers $\mathcal{O}_{K}$, where $\ell$ is a square free integer such that $\ell \equiv 3 \bmod 4$. The ring $\mathcal{O}_{K} /\left(2 \mathcal{O}_{K}\right)$ is equivalent to either the field of order 4 or a ring of order 4 depending on whether $\ell \equiv 3 \bmod 8$ or $\ell \equiv 7 \bmod 8$. We define bi-dimensional theta functions for the four cosets of $\mathcal{O}_{K} /\left(2 \mathcal{O}_{K}\right)$.

In the third section, we define codes over $\mathbb{F}_{4}$ and $\mathbb{F}_{2} \times \mathbb{F}_{2}$, the weight enumerators of a code, and recall the main result of [1]. We simplify the expressions for bidimensional theta series and prove Theorem 1 .

In the fourth section, we study families of codes corresponding to the same theta function. We call an acceptable theta series $\Theta(q)$ a series for which there exists a code $C$ such that $\Theta(q)=\Theta_{\Lambda_{\ell}}(C)(q)$. For any given $\ell$ and an acceptable theta series $\Theta(q)$ we can determine a family of symmetrized weight enumerators that correspond to $\Theta(q)$. For small $\ell$ this is a positive dimensional family, where the dimension is given by Theorem 2i). Hence, the example given in [1] is no surprise. For large $\ell$ (see Theorem 2ii)) this is a 0 -dimensional family of symmetrized weight enumerators that correspond to $\Theta(q)$. Therefore, the example that Chua provides cannot occur for larger $\ell$.

\section{IntRodUCTION TO LATTICES AND THETA FUNCTIONS}

Let $K$ be a number field and $\mathcal{O}_{K}$ be its ring of integers. A lattice $\Lambda$ over $K$ is an $\mathcal{O}_{K}$-submodule of $K^{n}$ of full rank. The Hermitian dual is defined by

$$
\Lambda^{*}=\left\{x \in K^{n} \mid x \cdot \bar{y} \in \mathcal{O}_{K} \text {, for all } y \in \Lambda\right\},
$$

where $x \cdot y:=\sum_{i=1}^{n} x_{i} y_{i}$. In the case that $\Lambda$ is a free $\mathcal{O}_{K}$-module, for every $\mathcal{O}_{K}$ basis $\left\{v_{1}, v_{2}, \ldots ., v_{n}\right\}$ we can associate a Gram matrix $\mathrm{G}(\Lambda)$ given by $G(\Lambda)=\left(v_{i} \cdot v_{j}\right)_{i, j=1}^{n}$ and the determinant $\operatorname{det} \Lambda:=\operatorname{det}(G)$ defined up to squares of units in $\mathcal{O}_{K}$. If $\Lambda=\Lambda^{*}$, then $\Lambda$ is Hermitian self-dual (or unimodular) and integral if and only if $\Lambda \subset \Lambda^{*}$. An integral lattice has the property $\Lambda \subset \Lambda^{*} \subset \frac{1}{\operatorname{det} \Lambda} \Lambda$. An integral lattice is called even if $x \cdot x \equiv 0 \bmod 2$ for all $x \in \Lambda$, and otherwise it is odd. An odd unimodular lattice is called a Type 1 lattice, and an even unimodular lattice is called a Type 2 lattice.

The theta series of a lattice $\Lambda$ in $K^{n}$ is given by $\Theta_{\Lambda}(\tau)=\sum_{z \in \Lambda} e^{\pi i \tau z \bar{z}}$, where $\tau \in H=\{z \in \mathbb{C}: \operatorname{Im}(z)>0\}$. Usually we let $q=e^{\pi i \tau}$. Then, $\Theta_{\Lambda}(q)=\sum_{z \in \Lambda} q^{z \bar{z}}$. 
The 1-dimensional theta series and its shadow are given by

$$
\theta_{3}(q):=\sum_{m \in \mathbb{Z}} q^{m^{2}}, \quad \theta_{2}(q):=\sum_{m \in \mathbb{Z}+1 / 2} q^{m^{2}} .
$$

Let $\ell>0$ be a square free integer and $K=Q(\sqrt{-\ell})$ be the imaginary quadratic field with discriminant $d_{K}$. Recall that $d_{K}=-\ell$ if $\ell \equiv 3 \bmod 4$ and $d_{K}=-4 \ell$ otherwise.

Let $\mathcal{O}_{K}$ be the ring of integers of $K$. The Hermitian lattice $\Lambda$ over $K$ is an $\mathcal{O}_{K}$-submodule of $K^{n}$ of full rank. Let $\ell \equiv 3 \bmod 4$ and let $d$ be a positive number such that $\ell=4 d-1$. Then, $-\ell \equiv 1 \bmod 4$. This implies that the ring of integers is $\mathcal{O}_{K}=\mathbb{Z}\left[\omega_{\ell}\right]$, where $\omega_{\ell}=\frac{-1+\sqrt{-\ell}}{2}$ and $\omega_{\ell}^{2}+\omega_{\ell}+d=0$. The principal norm form of $K$ is given by $Q_{d}(x, y)=\left|x-y \omega_{\ell}\right|^{2}=x^{2}+x y+d y^{2}$. Since $\ell \equiv 3$ $\bmod 4$, we can consider two cases:

(1) If $\ell \equiv 3 \bmod 8$, then $-\ell \equiv 5 \bmod 8$. Thus, the prime ideal $\langle 2\rangle \subset \mathbb{Z}$ lifts to a prime $2 \mathcal{O}_{K} \subset \mathcal{O}_{K}$. Since the ring of integers $\mathcal{O}_{K}$ is a Dedekind domain, $2 \mathcal{O}_{K}$ is a maximal ideal. Therefore $\mathcal{O}_{K} /\left(2 \mathcal{O}_{K}\right) \simeq \mathbb{F}_{4}$.

(2) If $\ell \equiv 7 \bmod 8$, then $-\ell \equiv 1 \bmod 8$. Then the prime ideal $\langle 2\rangle \in \mathbb{Z}$ splits in $K$. Therefore $2 \mathcal{O}_{K}$ splits in $\mathcal{O}_{K}$. Hence, $\mathcal{O}_{K} /\left(2 \mathcal{O}_{K}\right) \simeq \mathbb{F}_{2} \times \mathbb{F}_{2}$. In either case, a complete set of coset representatives is $\left\{0,1, \omega_{\ell}, 1+\omega_{\ell}\right\}$.

Let the following be the bi-dimensional theta series for the four cosets:

$$
\begin{aligned}
& A_{d}(q):=\Theta_{2 \mathcal{O}_{K}}(\tau)=\sum_{m, n \in \mathbb{Z}} q^{4 Q_{d}(m, n)}, \\
& C_{d}(q):=\Theta_{1+2 \mathcal{O}_{K}}(\tau)=\sum_{m, n \in \mathbb{Z}} q^{4 Q_{d}\left(m+\frac{1}{2}, n\right)}, \\
& G_{d}(q):=\Theta_{\omega_{\ell}+2 \mathcal{O}_{K}}(\tau)=\sum_{m, n \in \mathbb{Z}} q^{4 Q_{d}\left(m, n+\frac{1}{2}\right)}, \\
& H_{d}(q):=\Theta_{1+\omega_{\ell}+2 \mathcal{O}_{K}}(\tau)=\sum_{m, n \in \mathbb{Z}} q^{4 Q_{d}\left(m+\frac{1}{2}, n+\frac{1}{2}\right)} .
\end{aligned}
$$

Then we have the following lemma.

Lemma 1. Bi-dimensional theta series can be further expressed in terms of the standard one-dimensional theta series and its shadow:

$$
\begin{aligned}
& A_{d}(q)=\theta_{3}\left(q^{4}\right) \theta_{3}\left(q^{4 \ell}\right)+\theta_{2}\left(q^{4}\right) \theta_{2}\left(q^{4 \ell}\right), \\
& C_{d}(q)=\theta_{2}\left(q^{4}\right) \theta_{3}\left(q^{4 \ell}\right)+\theta_{3}\left(q^{4}\right) \theta_{2}\left(q^{4 \ell}\right), \\
& G_{d}(q)=H_{d}(q)=\frac{\theta_{2}(q) \theta_{2}\left(q^{\ell}\right)}{2} .
\end{aligned}
$$

Moreover,

$$
2 G_{d}(q)=A_{d}\left(q^{1 / 4}\right)-A_{d}(q)-C_{d}(q) .
$$

Proof. See [3] for details.

\section{Codes Over $\mathbb{F}_{4}$ AND $\mathbb{F}_{2} \times \mathbb{F}_{2}$}

Let $\mathbb{F}_{4}=\left\{0,1, \omega, \omega^{2}\right\}$, where $\omega^{2}+\omega+1=0$, be the finite field of four elements. The conjugation is given by $\bar{x}=x^{2}, x \in \mathbb{F}_{4}$. In particular $\bar{\omega}=\omega^{2}=\omega+1$. Let $R_{4}=\mathbb{F}_{2}+\omega \mathbb{F}_{2}$ where the new equation for $\omega$ being $\omega^{2}+\omega=0$. Notice that $R_{4}$ has two maximal ideals, namely $\langle\omega\rangle$ and $\langle\omega+1\rangle$. Furthermore, one can show that 
$R_{4} /\langle\omega\rangle$ and $R_{4} /\langle\omega+1\rangle$ are both isomorphic to $\mathbb{F}_{2}$. The Chinese remainder theorem tells us that $R_{4}=\langle\omega\rangle \oplus\langle\omega+1\rangle$. Therefore, $R_{4} \simeq \mathbb{F}_{2} \times \mathbb{F}_{2}$. The conjugate of $\omega$ is $\omega+1$. Let $\mathcal{R}$ be the field $\mathbb{F}_{4}$ if $\ell \equiv 3 \bmod 8$ or the ring $R_{4} \simeq \mathbb{F}_{2} \times \mathbb{F}_{2}$ when $\ell \equiv 7$ $\bmod 8$. A linear code $C$ of length $n$ over $\mathcal{R}$ is an $\mathcal{R}$-submodule of $\mathcal{R}^{n}$. The dual is defined as $C^{\perp}=\{u \in \mathcal{R}: u \cdot \bar{v}=0$ for all $v \in C\}$. If $C=C^{\perp}$, then $C$ is self-dual.

We define $\Lambda_{\ell}(C):=\left\{x \in \mathcal{O}_{K}^{n}: \rho_{\ell}(x) \in C\right\}$ where $\rho_{\ell}: \mathcal{O}_{K} \rightarrow \mathcal{O}_{K} / 2 \mathcal{O}_{k} \rightarrow \mathcal{R}$. In other words, $\Lambda_{\ell}(C)$ consists of all vectors in $\mathcal{O}_{K}^{n}$ which when taken $\bmod 2 \mathcal{O}_{K}$ componentwise are in $\rho_{\ell}^{-1}(C)$. The following is immediate.

Lemma 2. (1) $\Lambda_{\ell}(C)$ is an $\mathcal{O}_{K}$-lattice.

(2) $\Lambda_{\ell}\left(C^{\perp}\right)=2 \Lambda_{\ell}(C)^{*}$.

(3) $C$ is self-dual if and only if $\frac{\Lambda_{\ell}(C)}{\sqrt{2}}$ is self-dual.

Let $u=\left(u_{1}, u_{2}, \ldots, u_{n}\right) \in \mathcal{R}^{n}$ be a codeword and $\alpha \in \mathcal{R}$. Then the counting function $n_{\alpha}(u)$ is defined as the number of elements in the set $\left\{j: u_{j}=\alpha\right\}$. For a code $C$ we define the complete weight enumerator (cwe), symmetrized weight enumerator (swe) and Hamming weight enumerator $(W)$ to be

$$
\begin{aligned}
\text { cwe }_{C}(X, Y, Z, W) & :=\sum_{u \in C} X^{n_{0}(u)} Y^{n_{1}(u)} Z^{n_{\omega}(u)} W^{n_{1+\omega}(u)}, \\
\text { swe }_{C}(X, Y, Z) & :=\operatorname{cwe}_{C}(X, Y, Z, Z), \\
W_{C}(X, Y) & :=\operatorname{swe}_{C}(X, Y, Y) .
\end{aligned}
$$

Then we have the following.

Proposition 1. Let $\ell \equiv 3 \bmod 4, C$ be a linear code over $\mathcal{R}$, and $\frac{\Lambda_{\ell}(C)}{\sqrt{2}}$ be a Hermitian lattice constructed via the construction $A$. Then

$$
\theta_{\Lambda_{\ell}(C)}(\tau)=\operatorname{swe}_{C}\left(A_{d}(q), C_{d}(q), G_{d}(q)\right)
$$

where $A_{d}(q), C_{d}(q)$, and $G_{d}(q)$ are given as in (2.4).

For a proof of the above statement the reader can see [1]. From the definition of a one-dimensional theta series we have

$$
\theta_{2}(q)=2 q^{1 / 4} \sum_{i \in S} q^{i}, \quad \theta_{2}\left(q^{4}\right)=2 q \sum_{i: \text { odd }} q^{i^{2}-1}, \quad \theta_{3}\left(q^{4}\right)=1+2 q^{4} \sum_{i \in \mathbb{Z}^{+}} q^{4\left(i^{2}-1\right)},
$$

where $S=\left\{\frac{j^{2}-1}{4}: j \equiv 1 \bmod 2\right\}$. From $(2.4)$ we can write

$$
G_{d}(q)=\frac{\theta_{2}(q) \theta_{2}\left(q^{\ell}\right)}{2}=q^{\frac{(\ell+1)}{4}} \alpha_{1},
$$

where $\alpha_{1}=\sum_{i \in S} q^{i} \sum_{j \in S} q^{\ell j}$. Then,

$$
\begin{aligned}
A_{d}(q) & =\theta_{3}\left(q^{4}\right) \theta_{3}\left(q^{4 \ell}\right)+\theta_{2}\left(q^{4}\right) \theta_{2}\left(q^{4 \ell}\right) \\
& =\left(1+2 q^{4} \sum_{i \in \mathbb{Z}^{+}} q^{4\left(i^{2}-1\right)}\right)\left(1+2 q^{4 \ell} \sum_{j \in \mathbb{Z}^{+}} q^{4 \ell\left(j^{2}-1\right)}\right) \\
& +4 q^{\ell+1} \sum_{i: \text { odd }} q^{i^{2}-1} \sum_{j: \text { odd }} q^{\left(j^{2}-1\right) \ell} \\
& =\alpha_{2}+q^{\ell+1} \alpha_{3}+q^{4 \ell} \alpha_{4}
\end{aligned}
$$


where $\alpha_{2}, \alpha_{3}$ and $\alpha_{4}$ have the following forms:

$$
\begin{aligned}
& \alpha_{2}=1+2 q^{4} \sum_{i \in \mathbb{Z}^{+}} q^{4\left(i^{2}-1\right)}, \\
& \alpha_{3}=4 \sum_{i: \text { odd }} q^{i^{2}-1} \sum_{j: \text { odd }} q^{\left(j^{2}-1\right) \ell}, \\
& \alpha_{4}=2 \sum_{j \in \mathbb{Z}^{+}} q^{4 \ell\left(i^{2}-1\right)}\left(1+2 q^{4} \sum_{i \in \mathbb{Z}^{+}} q^{4\left(i^{2}-1\right)}\right) .
\end{aligned}
$$

Furthermore,

$$
\begin{aligned}
C_{d}(q) & =\theta_{2}\left(q^{4}\right) \theta_{3}\left(q^{4 \ell}\right)+\theta_{3}\left(q^{4}\right) \theta_{2}\left(q^{4 \ell}\right) \\
& =2 q \sum_{i: \text { odd }} q^{i^{2}-1}\left(1+2 q^{4 \ell} \sum_{i \in \mathbb{Z}^{+}} q^{4 \ell\left(i^{2}-1\right)}\right) \\
& +\left(1+2 q^{4} \sum_{i \in \mathbb{Z}^{+}} q^{4\left(i^{2}-1\right)}\right) 2 q^{\ell} \sum_{i: \text { odd }} q^{\left(i^{2}-1\right) \ell} \\
& =\alpha_{5}+q^{\ell} \alpha_{6}+q^{4 \ell+1} \alpha_{7},
\end{aligned}
$$

where $\alpha_{5}, \alpha_{6}$ and $\alpha_{7}$ have the following forms:

$$
\begin{aligned}
& \alpha_{5}=2 \sum_{i: \text { odd }} q^{i^{2}-1}, \\
& \alpha_{6}=2 \sum_{j \text { odd }} q^{\left(j^{2}-1\right) \ell}\left(1+2 q^{4} \sum_{i \in \mathbb{Z}^{+}} q^{4\left(i^{2}-1\right)}\right), \\
& \alpha_{7}=4 \sum_{i: \text { odd }} q^{i^{2}-1} \sum_{j \in \mathbb{Z}^{+}} q^{4 \ell\left(j^{2}-1\right)} .
\end{aligned}
$$

The next result shows that for large enough admissible $\ell$ and $\ell^{\prime}$ the theta functions $\Theta_{\Lambda_{\ell}}(C)$ and $\Theta_{\Lambda_{\ell^{\prime}}}(C)$ are virtually the same.

Theorem 3. Let $C$ be a code defined over $R$. For all admissible $\ell, \ell^{\prime}$ such that $\ell>\ell^{\prime}$, the following holds:

$$
\Theta_{\Lambda_{\ell}}(C)=\Theta_{\Lambda_{\ell^{\prime}}}(C)+\mathcal{O}\left(q^{\frac{\ell^{\prime}+1}{4}}\right)
$$

Proof. Let

$$
\operatorname{swe}_{C}(X, Y, Z)=\sum_{i+j+k=n} a_{i, j, k} \cdot X^{i} Y^{j} Z^{k}
$$

be a degree $n$ polynomial. Write this as a polynomial in $Z$. Then

$$
\text { swe }_{C}(Z)=\sum_{k=0}^{n} L_{k} Z^{k}=L_{0}+Z\left(\sum_{k=1}^{n} L_{k} Z^{k-1}\right) \text {. }
$$

Terms in $L_{0}$ are of the form of $a_{i, j} X^{i} Y^{j}$, where $i+j=n$. From the above we have

$$
\begin{aligned}
A_{d}(q)^{i} \cdot C_{d}(q)^{j} & =\left(\alpha_{2}+q^{\ell+1} \alpha_{3}+q^{4 \ell} \alpha_{4}\right)^{i} \cdot\left(\alpha_{5}+q^{\ell} \alpha_{6}+q^{4 \ell+1} \alpha_{7}\right)^{j} \\
& =(\text { terms independent from } \ell)+q^{\ell}(\cdots) .
\end{aligned}
$$

Also we have seen that $G_{d}(q)=q^{(\ell+1) / 4} \alpha_{1}$. This gives

$$
\begin{aligned}
\Theta_{\Lambda_{\ell}}(C) & =\text { swe }_{C}\left(A_{d}(q), C_{d}(q), G_{d}(q)\right) \\
& =(\text { terms independent from } \ell)+\mathcal{O}\left(q^{\frac{\ell+1}{4}}\right)
\end{aligned}
$$


Then the result follows.

Example 1. Let $C$ be a code defined over $R_{4}$ that has symmetrized weight enumerator

$$
\operatorname{swe}_{C}(X, Y, Z)=X^{3}+X^{2} Z+X Y^{2}+2 X Z^{2}+Y^{2} Z+2 Z^{3} .
$$

Then we have the following:

$$
\begin{aligned}
& \Theta_{\Lambda_{63}}(C)=1+6 q^{4}+12 q^{8}+8 q^{12}+12 q^{16}+6 q^{18}+48 q^{20}+30 q^{22}+\cdots, \\
& \Theta_{\Lambda_{79}}(C)=1+6 q^{4}+12 q^{8}+8 q^{12}+6 q^{16}+30 q^{20}+6 q^{22}+48 q^{24}+\cdots, \\
& \Theta_{\Lambda_{79}}(C)=\theta_{\Lambda_{63}}(C)+\mathcal{O}\left(q^{16}\right) .
\end{aligned}
$$

\section{A FAMily OF CODES CORRESPONDING TO THE SAME THETA FUNCTION}

If we are given the code over $\mathcal{R}$ and its symmetrized weight enumerator polynomial, then by (3.2) we can find the theta function of the lattice constructed from the code by using the construction $A$. Now, we would like to give a way to construct families of codes corresponding to the same theta function.

Let $\Theta(q)=\sum_{i=0}^{\infty} \lambda_{i} q^{i}$ be an acceptable theta series for level $\ell$ and

$$
f(x, y, z)=\sum_{i+j+k=n} c_{i, j, k} x^{i} y^{j} z^{k}
$$

be a degree $n$ generic ternary homogeneous polynomial. We want to find out how many polynomials $f(x, y, z)$ correspond to $\Theta(q)$ for a fixed $\ell$.

We have the following lemma.

Lemma 3. Let $C$ be a code of size $n$ defined over $\mathcal{R}$ and $\Theta(q)$ be its theta function for level $\ell$. Then, $\Theta(q)$ is uniquely determined by its first $\frac{n(\ell+1)}{4}$ coefficients.

Proof. Let $C$ be a code over $\mathcal{R}, \Theta(q)=\sum_{i=0}^{\infty} \lambda_{i} q^{i}$ be its theta series, $s=\frac{n(\ell+1)}{4}$ and

$$
f(x, y, z)=\sum_{i+j+k=n} c_{i, j, k} x^{i} y^{j} z^{k}
$$

be a degree $n$ generic ternary homogeneous polynomial. Find $A_{d}(q), C_{d}(q), G_{d}(q)$ for the given $\ell$ and substitute it in $f(x, y, z)$. Hence $f(x, y, z)$ is now written as a series in $q$. Recall that a generic degree $n$ ternary polynomial has $r=\frac{(n+1)(n+2)}{2}$ coefficients. So, the corresponding coefficients of the two sides of the equation are equal:

$$
f\left(A_{d}(q), C_{d}(q), G_{d}(q)\right)=\sum_{i=0}^{\infty} \lambda_{i} q^{i} .
$$

Consider the term

$$
c_{i, j, k}\left(\alpha_{2}+q^{\ell+1} \alpha_{3}+q^{4 \ell} \alpha_{4}\right)^{i}\left(\alpha_{5}+q^{\ell} \alpha_{6}+q^{4 \ell+1} \alpha_{7}\right)^{j}\left(q^{\frac{(\ell+1)}{4}} \alpha_{1}\right)^{k} .
$$

Then $c_{i, j, k}$ appears first as a coefficient of $q^{j+\frac{k(\ell+1)}{4}}$. For all such $j, k$ we have $j+\frac{k(\ell+1)}{4} \leq \frac{n(\ell+1)}{4}$. Consider the equations where $c_{i, j, k}$ appears first. This is a system of equations with $\leq \frac{(n+1)(n+2)}{2}$ equations. Let us denote this system of equations as $\Xi$. Solve this system for $c_{i, j, k}$. Hence, $c_{i, j, k}$ is a function of $\lambda_{0}, \ldots, \lambda_{s}$. For each $\mu>s, \lambda_{\mu}$ is a function of $c_{i, j, k}$ for $i, j, k=0, \ldots, n$, and therefore a rational function on $\lambda_{0}, \ldots, \lambda_{s}$. This completes the proof. 
Next we have the following theorem:

Theorem 2. Let $C$ be a code of size $n$ defined over $\mathcal{R}$ and $\Theta_{\Lambda_{\ell}}(C)$ be its corresponding theta function for level $\ell$. Then the following hold:

i) For $\ell<\frac{2(n+1)(n+2)}{n}-1$, there is a $\delta$-dimensional family of symmetrized weight enumerator polynomials corresponding to $\Theta_{\Lambda_{\ell}}(C)$, where $\delta$ $\geq \frac{(n+1)(n+2)}{2}-\frac{n(\ell+1)}{4}-1$.

ii) For $\ell \geq \frac{2(n+1)(n+2)}{n}-1$ and $n<\frac{\ell+1}{4}$, there is a unique symmetrized weight enumerator polynomial that corresponds to $\Theta_{\Lambda_{\ell}}(C)$.

Proof. We want to find out how many polynomials $f(x, y, z)$ correspond to $\Theta_{\Lambda_{\ell}}(C)$ for a fixed $\ell$. $\Theta_{\Lambda_{\ell}}(C)$ and $f(x, y, z)$ are defined as above. Consider the system of equations $\Xi$.

If $\frac{n(\ell+1)}{4}<r$, then our system has more variables than equations. Since the system is linear, the solution space is a family of positive dimension.

If $\frac{n(\ell+1)}{4} \geq r$, then for each equation in $\Xi$ (see the proof of the previous lemma) we have only one $c_{i, j, k}$ appearing for the first time. Otherwise suppose $c_{i, j, k}$ and $c_{i^{\prime}, j^{\prime}, k^{\prime}}$ appear for the first time in an equation of $\Xi$. Then $j+\frac{k(\ell+1)}{4}=j^{\prime}+\frac{k^{\prime}(\ell+1)}{4}$. This implies

$$
4\left(j-j^{\prime}\right)=\left(k^{\prime}-k\right)(\ell+1) .
$$

Without loss of generality, assume $k^{\prime} \geq k$. We can consider three cases.

Case 1: If $k^{\prime}-k \geq 2$, then from (4.1) we have $4 n\left(j-j^{\prime}\right)=n\left(k^{\prime}-k\right)(\ell+1)$ $\geq 4 r\left(k^{\prime}-k\right)$. Then we have $n\left(j-j^{\prime}\right) \geq(n+1)(n+2)$. Since $n \geq\left(j-j^{\prime}\right)$, we have a contradiction.

Case 2: If $k^{\prime}-k=1$, then by (4.1), $j-j^{\prime}=\frac{\ell+1}{4}$. Since $j-j^{\prime} \leq n$ and $\frac{\ell+1}{4}>n$, we get a contradiction.

Case 3: If $k^{\prime}-k=0$, then by (4.1) we have $j=j^{\prime}$. Hence $i=i^{\prime}$.

Notice that $c_{n, 0,0}$ is uniquely determined by the equation corresponding to the equation of the coefficient of $q^{0}$. Solve the system $\Xi$ in the order of the equation that corresponds to the power of $q$. We have a unique solution for $c_{i, j, k}$.

4.1. Families of codes of length 3. In this section we discuss the codes of length 3 for different levels $\ell$. Our main goal is to investigate the example provided in [1] and provide some computational evidence for the above two cases. We assume that the symmetrized weight enumerator polynomial is a generic homogenous polynomial of degree three.

Let $P(x, y, z)$ be a generic ternary cubic homogeneous polynomial given as below:

$$
\begin{aligned}
P(x, y, z) & =c_{1} x^{3}+c_{2} y^{3}+c_{3} z^{3}+c_{4} x^{2} y+c_{5} x^{2} z+c_{6} y^{2} x+c_{7} y^{2} z \\
& +c_{8} z^{2} x+c_{9} z^{2} y+c_{10} x y z .
\end{aligned}
$$

Assume that there is a code $C$, of length 3 , defined over $\mathcal{R}$ such that $s w e_{C}(x, y, z)$ $=P(x, y, z)$. First we have to fix the level $\ell$. When we fix the level, we can find $A_{d}(q), C_{d}(q), G_{d}(q)$. By equating both sides of

$$
p\left(A_{d}(q), C_{d}(q), G_{d}(q)\right)=\sum_{i=0}^{\infty} \lambda_{i} q^{i}
$$


we can get a system of equations. When $\ell=7$, we are in the first case of the previous theorem. The system of equations is given by the following:

$$
\left\{\begin{array} { l } 
{ c _ { 1 } - \lambda _ { 0 } = 0 , } \\
{ 2 c _ { 4 } - \lambda _ { 1 } = 0 , } \\
{ 4 c _ { 6 } + 2 c _ { 5 } - \lambda _ { 2 } = 0 , } \\
{ 8 c _ { 2 } + 4 c _ { 1 0 } - \lambda _ { 3 } = 0 }
\end{array} \quad \left\{\begin{array}{l}
6 c_{1}+4 c_{8}+2 c_{5}+8 c_{7}-\lambda_{4}=0, \\
8 c_{4}+8 c_{9}+4 c_{10}-\lambda_{5}=0, \\
8 c_{5}+8 c_{3}+8 c_{7}+8 c_{8}+8 c_{6}-\lambda_{6}=0 .
\end{array}\right.\right.
$$

The solution for the above system is given by $c_{1}=\lambda_{0}, c_{4}=\frac{1}{2} \lambda_{1}$, and

$$
\begin{aligned}
c_{2} & =\frac{1}{2} \lambda_{1}+\frac{1}{8} \lambda_{3}-\frac{1}{8} \lambda_{5}+c_{9}, \quad c_{3}=\frac{3}{2} \lambda_{0}-\frac{1}{4} \lambda_{2}-\frac{1}{4} \lambda_{4}+\frac{1}{8} \lambda_{6}+c_{7}, \\
c_{5} & =-3 \lambda_{0}+\frac{1}{2} \lambda_{4}-4 c_{7}-2 c_{8}, \quad c_{6}=\frac{3}{2} \lambda_{0}+\frac{1}{4} \lambda_{2}-\frac{1}{4} \lambda_{4}+2 c_{7}+c_{8}, \\
c_{10} & =-\lambda_{1}+\frac{1}{4} \lambda_{5}-2 c_{9}
\end{aligned}
$$

where $c_{7}, c_{8}, c_{9}$ are free variables. By giving different triples $\left(c_{7}, c_{8}, c_{9}\right)$, we can construct different polynomials $P(x, y, z)$ for the same $\sum_{i=0}^{\infty} \lambda_{i} q^{i}$.

Consider the following theta function. From [1] there are two nonisomorphic codes that give this theta function for level $\ell=7$ :

$$
\theta_{\sqrt{2} \mathcal{O}_{K}^{3}}=1+6 q^{2}+24 q^{4}+56 q^{6}+114 q^{8}+168 q^{10}+280 q^{12}+294 q^{14}+\cdots
$$

For this particular theta function, we can rewrite the solution (Eq. (4.4)) as follows: $c_{1}=1, c_{2}=c_{9}, c_{3}=1+c_{7}, c_{4}=0, c_{5}=9-4 c_{7}-2 c_{8}, c_{6}=-3-2 c_{7}+c_{8}, c_{10}=-2 c_{9}$.

For the triple $(1,2,0)$ (resp., $(0,3,0))$ we get the symmetrized weight enumerator polynomial for the code $C_{3,2}\left(\right.$ resp. $\left.C_{3,3}\right)$. That is, swe $C_{3,2}(X, Y, Z)=X^{3}+X^{2} Z+$ $X Y^{2}+2 X Z^{2}+Y^{2} Z+2 Z^{3}$ (resp., swe sw,3 $_{C_{3,3}}(X, Y, Z)=X^{3}+3 X^{2} Z+3 X Z^{2}+Z^{3}$ ), where $C_{3,2}$ and $C_{3,3}$ are given by

$$
\begin{aligned}
& C_{3,2}=\omega\langle[0,1,1]\rangle+(\omega+1)\langle[0,1,1]\rangle^{\perp}, \\
& C_{3,3}=\omega\langle[0,0,1]\rangle+(\omega+1)\langle[0,0,1]\rangle^{\perp} .
\end{aligned}
$$

When $\ell=15$, we are in the second case of the above theorem. The system of equations is as follows:

$$
\left\{\begin{array} { l } 
{ c _ { 1 } - \lambda _ { 0 } = 0 , } \\
{ 2 c _ { 4 } - \lambda _ { 1 } = 0 , } \\
{ 4 c _ { 6 } - \lambda _ { 2 } = 0 , } \\
{ 8 c _ { 2 } - \lambda _ { 3 } = 0 , } \\
{ 6 c _ { 1 } + 2 c _ { 5 } - \lambda _ { 4 } = 0 , }
\end{array} \quad \left\{\begin{array}{l}
8 c_{4}+4 c_{10}-\lambda_{5}=0, \\
2 c_{5}+8 c_{7}+8 c_{6}-\lambda_{6}=0, \\
4 c_{8}+8 c_{7}+12 c_{1}+8 c_{5}-\lambda_{8}=0, \\
10 c_{4}+8 c_{9}+8 c_{10}-\lambda_{9}=0, \\
8 c_{7}+8 c_{5}+12 c_{8}+8 c_{3}+8 c_{1}-\lambda_{12}=0 .
\end{array}\right.\right.
$$

Each $c_{i}$ appears first in exactly one equation. For example, consider the seventh equation. $c_{7}$ is the only variable that appears first in the seventh equation. Solve the system in the given order. The solution for the above system is given by: $c_{1}=\lambda_{0}, c_{2}=\frac{1}{8} \lambda_{3}, c_{4}=\frac{1}{2} \lambda_{1}, c_{6}=\frac{1}{4} \lambda_{2}$, and

$$
\begin{aligned}
c_{3} & =-\lambda_{0}-\frac{1}{2} \lambda_{2}+\frac{3}{4} \lambda_{4}+\frac{1}{4} \lambda_{6}-\frac{3}{8} \lambda_{8}+\frac{1}{8} \lambda_{12}, & c_{5} & =-3 \lambda_{0}+\frac{1}{2} \lambda_{4}, \\
c_{7} & =\frac{3}{4} \lambda_{0}-\frac{1}{4} \lambda_{2}-\frac{1}{8} \lambda_{4}+\frac{1}{8} \lambda_{6}, & c_{9} & =\frac{3}{8} \lambda_{1}-\frac{1}{4} \lambda_{5}+\frac{1}{8} \lambda_{9}, \\
c_{8} & =\frac{3}{2} \lambda_{0}+\frac{1}{2} \lambda_{2}-\frac{3}{4} \lambda_{4}-\frac{1}{4} \lambda_{6}+\frac{1}{4} \lambda_{8}, & c_{10} & =-\lambda_{1}+\frac{1}{4} \lambda_{5} .
\end{aligned}
$$


We have a unique solution. This implies that two nonequivalent codes cannot give the same theta function for $\ell=15$ and $n=3$.

\section{Concluding Remarks}

The main goal of this paper was to find out how theta functions determine the codes over a ring of size 4 . First we have shown how the theta functions of the same code $C$ differ for different levels $\ell$. The first $\frac{\ell+1}{4}$ terms of the theta functions for levels $\ell$ and $\ell^{\prime}$ are the same, where $\ell^{\prime} \geq \ell$.

In [1], two nonisomorphic codes that give the same theta function for level $\ell=7$ but not under higher level constructions are given. We justified the reason why we don't have a similar situation for higher level constructions. In this note we have addressed a method that we can use for finding a family of polynomials that correspond to a given acceptable theta series for some fixed level $\ell$. We have studied two cases depending upon $\ell$ that give either a positive dimensional family of polynomials or a unique polynomial.

\section{REFERENCES}

[1] K. S. Chua, Codes over $\mathrm{GF}(4)$ and $\mathbf{F}_{2} \times \mathbf{F}_{2}$ and Hermitian lattices over imaginary quadratic fields, Proc. Amer. Math. Soc., 133 (2005) no. 3, 661-670 (electronic). MR2113912 (2005i:11086).

[2] N. J. A. Sloane, Codes over GF(4) and complex lattices, J. Algebra, 52 (1978), no. 1, 168-181. MR0490436 (58:9782).

[3] J. H. Conway and N. J. A. Sloane, Sphere packings, lattices and groups, Second edition, Grundlehren der Mathematischen Wissenschaften [Fundamental Principles of Mathematical Sciences], vol. 290, Springer-Verlag, New York, 1993. MR1194619 (93h:11069)

[4] H. H. Chan, K. S. Chua and P. Solé, Seven-modular lattices and a septic base Jacobi identity, J. Number Theory, 99 (2003), no. 2, 361-372. MR1968458 (2003m:11102)

Department of Mathematics and Statistics, Oakland University, 368 Science and Engineering Building, Rochester, Michigan 48309.

E-mail address: shaska@oakland.edu

Department of Mathematics and Statistics, Oakland University, 368 Science and Engineering Building, Rochester, Michigan 48309

E-mail address: gwijesi@oakland.edu 